\title{
Un rythme d'écriture
}

À propos d'Assia Djebar et de ses archives. Un entretien avec Mireille Calle-Gruber

\section{Mireille Calle-Gruber et Hervé Sanson}

\section{OpenEdition}

\section{Journals}

Édition électronique

URL : http://journals.openedition.org/coma/593

DOI : $10.4000 /$ coma.593

ISSN : 2275-1742

\section{Éditeur}

Institut des textes \& manuscrits modernes (ITEM)

\section{Référence électronique}

Mireille Calle-Gruber et Hervé Sanson, « Un rythme d'écriture », Continents manuscrits [En ligne]

5 | 2015, mis en ligne le 15 octobre 2015, consulté le 02 mai 2019. URL : http:// journals.openedition.org/coma/593 ; DOI : 10.4000/coma.593

Ce document a été généré automatiquement le 2 mai 2019.

\section{(c) (i) $\odot$}

Continents manuscrits - Génétique des textes littéraires - Afrique, Caraîbe, dispora est mis à disposition selon les termes de la licence Creative Commons Attribution - Pas d'Utilisation

Commerciale - Pas de Modification 4.0 International. 


\title{
Un rythme d'écriture
}

\author{
À propos d'Assia Djebar et de ses archives. Un entretien avec Mireille \\ Calle-Gruber
}

\author{
Mireille Calle-Gruber et Hervé Sanson
}

\begin{abstract}
$* * *$
Mireille Calle-Gruber est professeur émérite de littérature et d'esthétique à l'Université Paris III-La Sorbonne nouvelle et écrivain. Parmi ses nombreuses publications, celles consacrées à Assia Djebar ont fait d'elle la spécialiste incontestée de cette œuvre: Assia Djebar ou la résistance de l'écriture. Regards d'un écrivain d'Algérie, chez Maisonneuve \& Larose, 2001; Assia Djebar, Ministère des Affaires étrangères, ADPF, 2006; Assia Djebar, nomade entre les murs... (dir.), chez Maisonneuve \& Larose, 2005 ; Assia Djebar, littérature et transmission (dir.) aux PSN, 2010.

À la lecture du volume rédigé par Mireille Calle-Gruber pour l'ADPF, Assia Djebar réagissait ainsi dans un courrier électronique adressé à un tiers : « Je viens de lire le texte de Mireille... qui m'a émue jusqu'aux larmes. Non par vanité ou satisfaction d'auteur, mais parce que ce texte magnifique - et si «pointu» - m'a remise, par la mémoire dans l'exaltation qui était mienne quand j'écrivais ce texte (... il y a déjà 15 ou 16 ans...) et tu diras à Mireille qu'elle m'a replacée émotionnellement dans mon écriture de cette époque (89-90). Elle m'a donc redonné force - après le $4^{\mathrm{e}}$ du quatuor, de finir cet ensemble de 3 sur l'Islam... Elle est plus que commentatrice; elle m'épaule, elle m'aiguillonne, elle me remet à la source même de mon écriture d'alors, mais parce qu'elle est écrivain, en même temps que critique, qu'elle est solidaire et sœur vraiment dans la poésie... »
\end{abstract}

$* * *$

HERVÉ SANSON : Mireille, tu es l'une des grandes spécialistes de l'œuvre d'Assia Djebar, dont tu as été l'amie durant une vingtaine d'années. Aujourd'hui, nous nous retrouvons pour 
évoquer sa méthode de travail, et son rapport aux manuscrits. Ma question sera donc simple : quel est, d'après ce que tu en sais, l'état de son fonds et de quoi se compose-t-il ?

Mireille Calle-Gruber : Peut-on vraiment parler de fonds pour Assia? C'est bien le problème: nous avons souvent parlé, elle et moi, de ses archives, il y a plusieurs années; elle aurait souhaité que nous parvenions toutes les deux à créer un fonds de son vivant, afin de réunir les choses et d'aider sa fille Jalila qui est son héritière et son ayant droit, à en assurer la transmission. Mais par ailleurs, Assia n'avait pas très envie de se lancer dans des démarches, une administration, une gestion des choses. Tout cela lui pesait. Peut-être aussi qu'elle n'avait pas du tout envie de penser à l'après-Assia, et c'est vrai que moi non plus je n'avais pas très envie d'y penser. C'est donc resté en l'air. Je me suis dit que nous avions le temps, qu'on en reparlerait... Je lui avais donné mon accord, lui disant que je serais très heureuse de l'épauler et de donner du rayonnement à son œuvre que j'aime profondément. C'était bien avant son élection à l'Académie française qu'elle m'avait exprimé ce désir que je m'occupe de son œuvre après elle...

HeRVÉ SANSON : donc au début des années 2000...

Mireille Calle-Gruber : Fin des années quatre-vingt-dix, début des années 2000. Elle pensait qu'il fallait réunir les choses. Sans doute du fait qu'elle partait aux Etats-Unis, se sentait-elle plus dispersée. Jusque là, elle était entre l'Algérie et la France, tout d'un coup, la vie prenait des dimensions moins maîtrisables. Ce fut d'abord Bâton Rouge et Louisiana State University, puis il y eut New York qu'elle aimait, mais elle perdait un peu ses repères dans ces espaces américains, et elle avait besoin je crois d'abriter son œuvre dans un lieu défini, et de composer son archive dans l'amitié pour adoucir le côté post-mortem... Qu'en est-il aujourd'hui ? J'ai vu chez elle ses cahiers. Elle m'a montré dans son bureau, qui était un immense bazar, des rangées de cahiers sur lesquels elle avait écrit ses brouillons, pris des notes, et elle les appelait les « livres de brouillons »; c'est ainsi que je les ai désignés dans le volume du Ministère des Affaires étrangères Assia Djebar (La Documentation française, 2006), où deux pages du Blanc de l'Algérie sont reproduites. Elle avait une ample écriture, elle pouvait remplir comme ça très librement et un peu en biais la page, elle n'essayait pas de faire un texte... Ainsi était le début je crois, avec des plans, elle aimait faire des sortes de structures, avec des motsclefs, et des séquences numérotées. C'est ainsi qu'elle démarrait un livre. Ensuite, lorsque ça commençait à prendre corps, elle écrivait à la main, elle écrivait sur des feuillets A4 à la main toujours, et ce n'était pas elle qui saisissait mais une assistante. Puis elle retravaillait sur le tirage papier. Alors le fonds? Comme je le dis, il n'y en a pas. Tout reste à faire... 


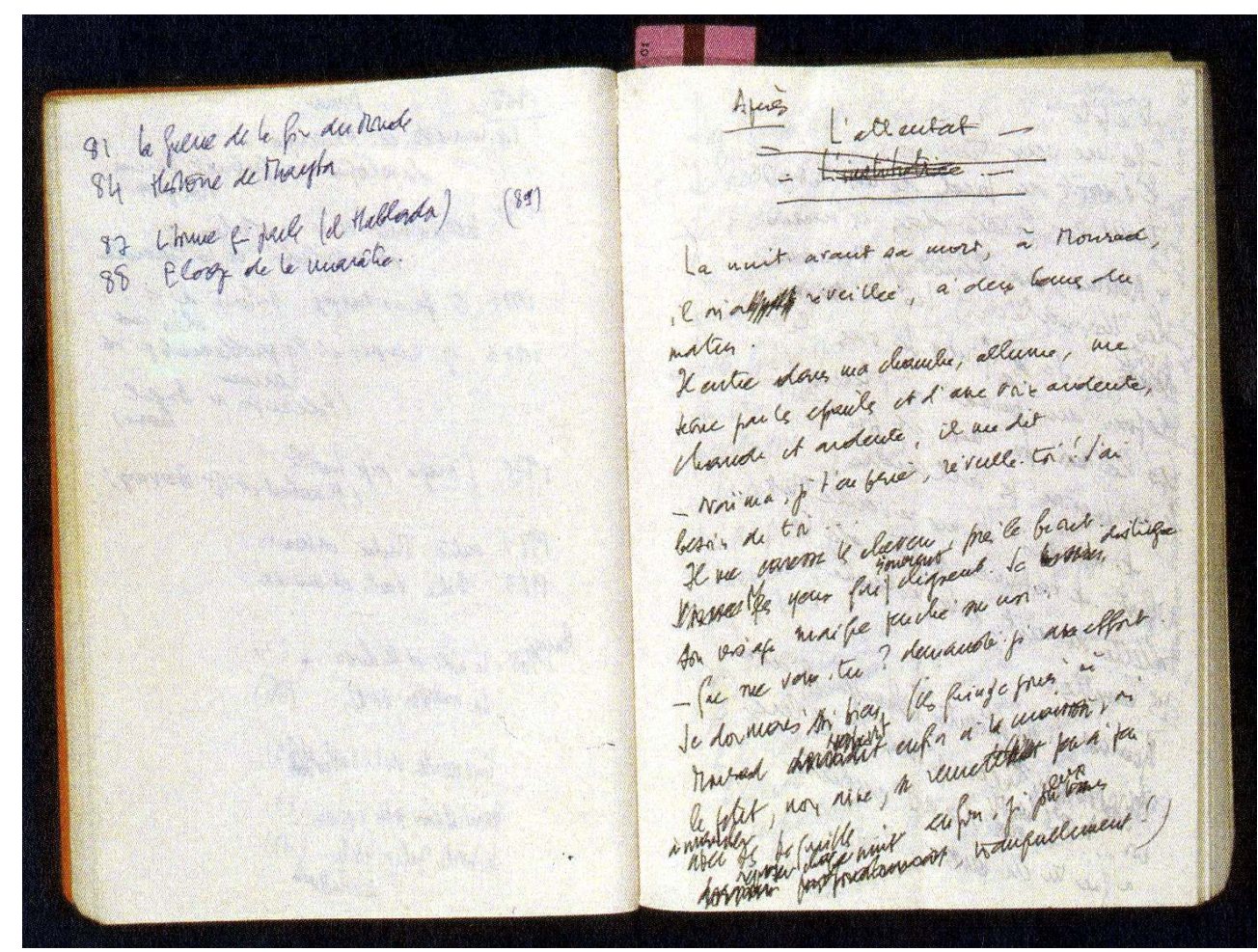

Reproduction avec l'aimable autorisation de M. Calle-Gruber qui avait été autorisée à publier cette image par A. Djebar elle-même.

HERVÉ SANSON : Tout le travail est à venir.

Mireille Calle-Gruber : A ma connaissance, elle n'a rien déposé nulle part, il a été question aussi qu'elle dépose des manuscrits dans les universités américaines qui la sollicitaient, mais cela lui demandait un travail de collation qu'elle n'a pas effectué. Je pense que la plupart de ses archives sont chez elle, puisque même ce qui était aux EtatsUnis a été re-déménagé à Paris, lorsqu'elle est rentrée... Nous sommes devant une stratification de temps d'écritures, il va falloir beaucoup de patience. C'est la même chose pour ses films, c'est peut-être encore pire, je crois qu'elle a une copie de chaque, et c'est tout. Elle n'a jamais pris le temps de démêler la question des droits et des copies avec l'Etat algérien. La situation semble très compliquée. Lorsqu'elle est venue en Allemagne, à l'Institut français de Heidelberg, puis au Canada à Queen's University où je l'avais invitée, elle est arrivée chaque fois avec sa bobine sous le bras...

HERVÉ SANSON : Donc une des premières tâches urgentes, ce serait effectivement de sauvegarder, de repiquer...

Mireille Calle-Gruber : déjà il faudrait faire un inventaire des pièces d'archives, un état des lieux, et sérier les questions juridico-politiques ... Je pense qu'une partie est bien repérable, en tout cas pour les livres à partir des années quatre-vingt. Les cahiers sont reliés, chacun constitue un ensemble...

HERVÉ SANSON : Ces cahiers, tu les as un peu regardés?...

Mireille Calle-Gruber : Elle m'en avait prêté certains afin que je numérise quelques pages pour publication: des nouvelles d'Oran, langue morte, "L'Attentat », par exemple ; un cahier portant sur Le Blanc de l'Algérie ; des notes générales... 
Fig. 2 Notes générales du Blanc de l'Algérie

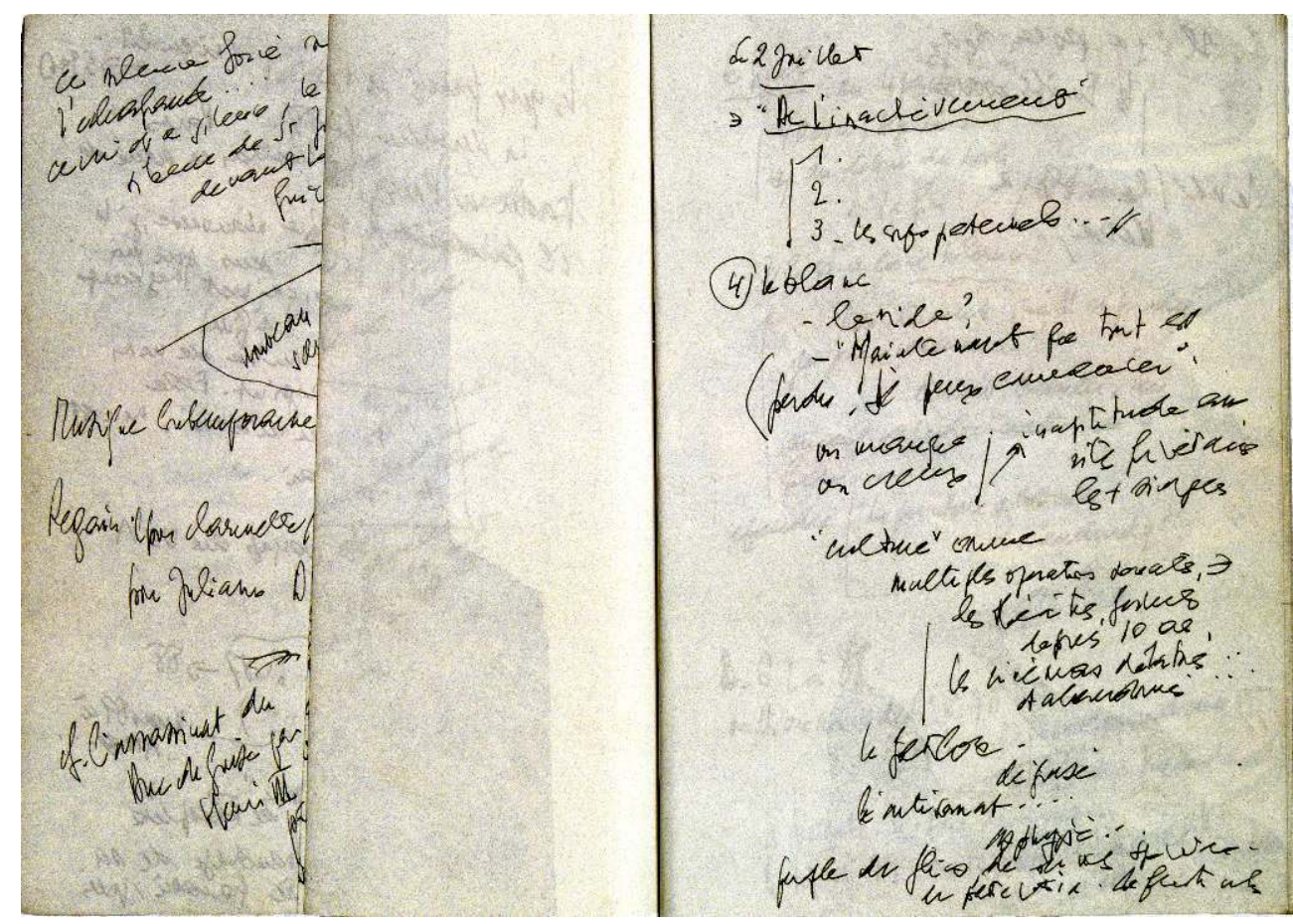

Reproduction avec l'aimable autorisation de M. Calle-Gruber qui avait été autorisée à publier cette image par A. Djebar elle-même.

HERVÉ SANSON : ça balaie l'ensemble de l'œuvre? A-t-elle a pu laisser des choses en Algérie? des manuscrits...

Mireille Calle-Gruber : Je ne pense pas. Il est vrai qu'il y a les tout premiers ouvrages dont je ne sais si elle a conservé les manuscrits. Je veux parler des romans repris par Actes sud, Les Alouettes naïves par exemple. Mais où en Algérie? Elle n'y avait plus d'appartement ni de logement depuis longtemps ...

HERVÉ SANSON : A priori c'est centralisé à Paris...

Mireille Calle-Gruber : Tout devrait être dans l'appartement de Paris où elle déposait ses affaires au fur et à mesure de ses passages et de ses séjours intermittents. C'est un lieu très... plein, tu vois, en chantier, comme quand on travaille et qu'on s'étale partout. Ça s'entassait. Parfois nous commencions à remuer les choses dans son bureau, et affleuraient des traces d'époques antérieures, de vies antérieures, c'était étonnant. Assia n'était pas quelqu'un qui jetait, elle gardait tout, on devrait tout trouver chez elle.

HeRVÉ SANSON : Oui, et comme tu le disais toi-même, on peut imaginer peut-être que les toutes premières œuvres... en plus il faut resituer l'époque, la guerre, un moment elle a suivi son mari, ils étaient plus ou moins dans la clandestinité, ou la semi-clandestinité, j'imagine que les choses ont pu se disperser...

Mireille Caller-Gruber : Ces manuscrits-là ont pu se perdre, oui. Se disperser, être détruits. C'est possible. Elle était très jeune, on ne pense pas "archives " quand on a dix-huit, dix-neuf ans, qu'on écrit dans une sorte d'urgence, qu'il y a en effet la guerre, les voyages, la clandestinité... Lorsqu'elle publie Femmes d'Alger dans leur appartement aux éditions des Femmes, c'est un nouveau départ littéraire, et la conscience de construire une œuvre organique. Elle projette des ensembles, le Quatuor algérien, la trilogie sur l'Islam. Alors les manuscrits deviennent une mémoire qui s'articule. 
HERVÉ SANSON : Enchaînons sur la question suivante. Tu as répondu à la question de savoir si elle avait conservé systématiquement ses manuscrits, les avant-textes de tous ses ouvrages... ou seulement les dernières œuvres... On peut se pencher maintenant plus spécifiquement sur sa méthode de travail. Tu as commencé à y répondre, tu as dit qu'elle écrivait à la main, qu'elle commençait par écrire à la main, cela c'était la première étape, et ensuite elle faisait mettre au net ce texte, elle avait donc une secrétaire...

Mireille Calle-Gruber : Elle travaillait avec ses étudiants, ses assistants de recherche, puisqu'elle enseignait à l'université en même temps.

Hervé SANSON : donc c'étaient ses assistants, assistantes, qui mettaient le texte au propre, qui le saisissaient...

Mireille Calle-Gruber : Lorsque je l'ai connue, c'était ainsi et ça a été ainsi jusqu'à la fin.

Hervé Sanson : D'accord. Et donc toujours sur cette question de la méthode de travail, toi qui l'as très bien connue, est-ce qu'elle avait une discipline, plus ou moins stricte, à laquelle elle se soumettait? ou bien écrivait-elle selon l'impulsion du moment?

Mireille Calle-Gruber : Quand elle était dans un livre, elle y était complètement. Absorbée et débordante. Comme tu sais, elle marchait beaucoup. Assia marchait dans la ville. C'était son luxe de femme libre. Elle écrivait aussi en marchant, ça s'écrivait, pas dans sa tête mais dans son corps, ça venait imprimer son corps. En marchant, elle trouvait des rythmes comme cela. Elle dansait l'écriture avant de la coucher sur la page. Et puis elle écrivait à tout moment, elle oubliait de manger, elle n'avait pas d'horaires, elle était dans cette sorte de débordement de l'écriture... Ce n'était pas quelqu'un qui dit « je vais travailler de neuf heures à midi... »

HERVÉ SANSON : Oui, c'est cela qui est intéressant aussi. Parce qu'évidemment il y a toute une mythologie de l'écrivain à sa table de travail très courante... avec effectivement le fameux conseil de l'écrivain, de l'aîné «Installez-vous à votre table à huit heures, et ce jusqu'à midi... » C'est ce que l'on trouve le plus souvent, et elle, elle n'est pas du tout dans cette économie...

Mireille Calle-Gruber : Pas du tout. C'est un écrivain nomade. Marcher dans les rues de Paris, ou de New York, c'était très important pour elle, le rythme de la marche c'était aussi un rythme d'écriture... un rythme de langue qui lui arrivait de très loin, de son héritage andalou, de ses ancêtres berbères, des récits des gynécées. Il faut relire Ces voix qui m'assiègent, elle l'explicite très bien.

HERVÉ SANSON : Oui, donc pas d'heure et pas d'espace privilégié...

Moi, ce que j'en sais, et bien sûr j'en sais moins que toi, c'est qu'elle pouvait écrire dans un café...

Mireille Calle-Gruber : C'est vrai, elle aimait les cafés, c'était plutôt des lieux de haltes, éventuellement de prise de notes, et de rêverie. Il y a autre chose : Assia écrit à partir de ce qu'on lui raconte. Elle aimait entendre les récits des Algériens qui revenaient en France, l'un racontait ce que sa mère lui avait raconté, ce que la grand-mère avait raconté à la mère, elle était celle qui savait écouter et recevoir les sagas des autres. Beaucoup de ses livres sont nourris de ces micro-récits, d'une mémoire orale pleine d'accents idiomatiques. Ce rapport à l'oralité est un déclencheur d'écriture: elle transcrit, elle est comme elle le dit souvent la scripteuse, la passeuse... et son travail consiste à en faire un texte. La Disparition de la langue française c'est l'histoire qu'un ami lui a racontée... La plupart des nouvelles d'Oran, langue morte partent de récits vécus, si l'on peut dire, mais ce n'est pas tant le vécu que le récit déjà transmis. Sa transmission 
dans un langage singulier. Elle recueille. A cet égard, L'Amour, la fantasia est emblématique : la troisième partie reprend et «traduit » les entretiens qu'elle a faits pour son film La Nouba des femmes du mont Chenoua, dans la tribu de sa mère. Elle en fait des micro-récits de témoignages en langue vernaculaire, qui enclenchent la narration romanesque. Son écriture est déjà une phase seconde à partir de notes et de choses qu'elle a écoutées, qui lui viennent de son passé, de son pays, de sa langue, et qui la relient à une antériorité. L'écriture sur papier est déjà seconde. D'abord affleure une sorte d'archive orale dont elle se fait le réceptacle, le corps. Je voudrais dire aussi quelque chose qui m'a beaucoup touchée : on se parlait la nuit, surtout lorsqu'elle était à New York, du fait du décalage horaire. Elle m'appelait, notamment pour Nulle part dans la maison de mon père, qui est un livre qu'elle a beaucoup souffert. Assia était un écrivain qui pleure en écrivant, qui vivait cette écriture d'une manière déchirée, déchirante, tu vois? Qui se posait des questions, qui était en colère, qui avait tous les états de corps et d'âme quand elle écrivait. Et moi je recevais cela par téléphone, c'était étrange, ça faisait partie de son rapport à l'oralité. Elle pouvait parler - moi par exemple je n'arrive pas à parler de ce que j'écris au moment où je l'écris, tant que ce n'est pas écrit je ne sais pas, j'ai besoin de la lettre, d'écrire pour savoir. Elle, elle avait besoin de cette oralité, c'était une autre manière de peser et de penser l'écriture, de la travailler.

HERVÉ SANSON : Oui, d'ailleurs tu parlais de téléphone effectivement - on connaît ce texte court d'Assia, cette nouvelle qui s'appelle «L'Eplorée »...

Mireille Calle-Gruber : où le fil du téléphone c'est aussi le fil narratif, et le fil d'écriture...

HERVÉ SANson : C'est très intéressant effectivement, le livre dans la tête avant d'arriver au papier, et puis surtout cette marche, ce rythme associé à la marche, c'est cela que je trouve très intéressant et surtout très singulier... Alors, on revient aux manuscrits, et plus précisément au rapport aux manuscrits. Quel rapport, selon ce que tu en sais, Assia entretenait-elle avec ses brouillons, ses manuscrits, ses avant-textes?

Mireille Calle-Gruber : Je voyais Assia comme une nomade qui n'est pas tellement attachée aux choses. Elle était dans l'appartement de Paris, elle était aussi bien dans l'appartement de New York, au fond elle ne cherchait pas vraiment à s'installer quelque part, à Bâton Rouge elle n'était pas du tout installée. J'ai été étonnée de voir qu'elle avait gardé ses cahiers. Je me suis dit, alors quand même, vis-à-vis de l'écrit, il y a un attachement. Cependant, il n'était pas des plus forts, ni des plus fétichistes. J'avais toujours l'impression qu'Assia allait à la perte. Elle pouvait tout perdre, au fond ça n'avait pas d'importance. Elle était dans une sorte de devenir, d'ouverture à ce qui vient ; le manuscrit pour elle n'avait pas, je pense, une valeur fondamentale.

HERVÉ SANSON : Oui, elle n'était pas dans cette sorte de fétichisation... Mais tout de même le fait qu'elle les ait conservés....

Mireille Calle-Gruber : Elle les a gardés. Oui, j'étais étonnée, cela signifie quelque chose. Mais elle-même ne cessait de redécouvrir avec étonnement ce qu'elle avait écrit, ou qu'elle avait perdu, ou qu'elle ne pensait pas avoir écrit, ou qu'elle n'avait jamais retrouvé. Tu vois, elle était dans une dimension d' « acte manqué » de l'écriture... Peutêtre aussi par pudeur, parce qu'elle avait le sentiment de « s'exposer ». Aussi parce que ce qu'elle écrivait était transgressif aux yeux de la société, de la famille, de la religion. Elle n'était pas dans la thésaurisation du manuscrit, si tu veux. C'était une écriture généreuse, qui est une façon d'être à la vie, à la vie à la mort, à la vie à la perte... Elle ne 
veillait pas sur ses manuscrits comme sur une sorte de trésor. Pas d'attachement viscéral. Elle n'était pas du tout dans cette économie-là.

HERVÉ SANSON : Et en même temps, je me demande... cela fait écho en moi avec d'autres choses, je pense à d'autres écrivains algériens où il y a un peu ce que tu décris, ce rapport... les manuscrits existent, ils les ont quand même gardés, et en même temps il y a un rapport assez détaché aux manuscrits. On connaît tous Kateb et sa trajectoire ; même Dib, s'il a tout gardé systématiquement, il y a cette première période qu'il a finalement laissée en Algérie, et c'est ainsi... Je pense beaucoup à Sénac par exemple, qui pouvait offrir à ses amis un poème - il était très connu pour ça - et en même temps quand on va à la BNA, j'ai vu le fonds Sénac -, tout a été gardé, c'est un fonds très important, tous ses livres, il a quand même gardé les choses dans une grande mesure...

Mireille Calle-Gruber : Assia n'aurait jamais offert ses manuscrits, elle ne pensait pas que cela pouvait avoir de la valeur.

HeRVÉ SANSON : Oui, alors quand je disais que Sénac offrait, c'était plus dans l'idée qu'il venait de composer un poème, il offrait le poème du matin... ce n'était pas le manuscrit en tant que tel.

Mireille Calle-Gruber : Par contre, elle faisait un travail d'historienne, elle était historienne. Elle prenait beaucoup de notes, faisait des photos. A-t-elle gardé ses notes de lecture ? Je ne sais pas. Tous ses livres ont commencé par la lecture, des recherches en bibliothèque, le séjour dans les bibliothèques était très important.

HERVÉ SANSON : Oui, tu sais aussi bien que moi que pour les généticiens, les brouillons... toute la phase préparatoire est très importante...

Mireille Calle-Gruber : Toute la phase préparatoire était très importante pour elle. Elle est allée consulter les fonds d'archives pour l'histoire de l'Algérie, l'histoire de la colonisation. En ce qui concerne Loin de Médine et Filles d'Ismaël, elle se faisait aider pour le déchiffrement des textes anciens et de l'arabe classique. Quant au livre en cours, Les larmes d'Augustin, qui n'est pas terminé, elle a amassé une riche documentation : sur les discours d'Augustin, notamment à Césarée, mais aussi sur l'histoire de son arrièregrand-père qui était au Vietnam et donc sur une histoire de la colonisation en amont de celle de l'Algérie. Je vois donc deux genres de manuscrit: l'archive orale qui prend forme progressivement dans le corps écrit, et tout le travail documentaire préalable à partir de textes. Deux façons d'archives différentes.

HERVÉ SANSON : On peut maintenant aborder une question qui est peut-être très spécifique à Assia, qui est le souci de composition de ses ouvrages...

Mireille Calle-Gruber : Pour le dernier roman inachevé Les larmes d'Augustin, elle a écrit le texte, elle devait me le passer, mais ne l'a finalement pas envoyé (elle me faisait volontiers lire ses textes en cours). Elle ne l'a pas envoyé, car elle n'était pas satisfaite, elle disait qu'elle n'avait pas trouvé la composition. Pour elle c'était ce qui importait. Cela va poser des problèmes : est-ce qu'il est publiable comme ça ? Est-ce que ce n'est pas trahir son exigence d'architecture? C'est plus qu'une structure, c'est une architecture, une symphonie, il y a quelque chose de très musical dans les compositions d'Assia. Pour les films également : le premier est construit sur la forme de la nouba. Je me demande jusqu'à quel point on peut publier dans cet état non abouti à ses yeux. Tu le sais, elle a écrit beaucoup de livres entretemps, mais celui-là, ce quatrième du quatuor, elle n'arrivait pas à le porter à terme parce qu'elle n'arrivait pas à trouver la composition. 
HERVÉ SANSON : Et moi je me souviens d'un entretien qu'elle m'avait accordé alors que j'étais tout jeune étudiant, dans lequel elle me parlait en effet du temps que cela lui avait pris pour trouver l'architectonique de L'Amour, la fantasia. Elle y avait passé deux ans...

Mireille Calle-Gruber : Oui, c'est extrêmement beau, extrêmement subtil... Elle fait entendre des voix, des intensités, un chant choral, elle a le sens phonique des langues. À mon avis il vaudrait mieux publier cet écrit comme un travail en cours, un travail génétique, le mettre dans une collection d'archives, et l'entourer de ses notes préparatoires, en faire un dossier.

HERVÉ SANSON : Tout à fait. Je pense que c'est le respect le plus grand que l'on pourrait avoir pour elle... C'est dommage que cela se perde, ou que cela n'émerge jamais, mais il faut trouver l'approche juste, la forme juste... Et donc - tu y as déjà répondu en partie tout à l'heure - mais toujours en restant focalisé sur les manuscrits, tu as vu quelques-uns de ses cahiers, tu m'as parlé tout à l'heure de ces plans qu'elle faisait... dans ses manuscrits, on voit un peu ce souci de composition ?...

Mireille Calle-Gruber : On voit ce souci : elle met des mots, des flèches, opère des articulations... Cela, c'est le début. Après, lorsque l'ensemble prend de l'ampleur, qu'elle a écrit un texte à ses yeux trop linéaire, qui ne résonne pas, elle cherche des rythmes, des résonances, des échos, et compose au son. Elle est dans une esthétique araboandalouse, dans quelque chose de l'ordre du chant, de la poésie. Il y a aussi dans ses livres une langue française habitée des chants et transes transmis par les femmes. Toute une culture au féminin.

HERVÉ SANSON : Oui, et tout le plan musical... Elle travaille à l'oreille.

Mireille Calle-Gruber: C'est à l'oreille et davantage: elle travaille de grands ensembles, il faut que des fils traversent, qu'il y ait des courts-circuits, des chocs émotionnels. Question de rythme mais aussi de temporalité. On n'est plus dans la chronologie, ni la linéarité, on n'est plus dans le raisonnement. C'est le règne des analogies et de l'arabesque.

HERVÉ SANSON : Oui, des analogies temporelles... Elle travaille à la fois le macroscopique et le microscopique. Elle fait travailler les deux ensemble.

Mireille Calle-Gruber : Voilà, par analogies et par translations...

HeRvÉ SANSON : Et là j'en reviens à ma «mythologie d'écrivain » dont je parlais tout à l'heure... On voit toujours certains écrivains nous parler des canevas qu'ils mettent en place, où c'est très préparé en amont, et puis d'autres qui écrivent à l'aveugle, qui commencent l'écriture et ne savent pas où cela les mène, et Assia elle est un peu entre les deux, elle est dans une autre voie... Elle réajuste continûment...

Mireille Calle-Gruber : Absolument. Il faut avoir à l'esprit qu'Assia est quelqu'un de très visuel, c'est-à-dire qu'en même temps qu'elle écrit ou lit, elle « voit » les choses... Quand j'ai écrit mon roman Tombeau d'Akhnaton', elle a beaucoup aimé ce livre, elle m'a parlé longtemps au téléphone, car j'évoque dans ce livre le cinéaste égyptien Shâdi Abd As-Salâm qui a fait des films magnifiques et n'a pas eu le temps de tourner son Akhnaton . Elle était bouleversée car elle l'avait connu. Elle a voulu faire un film avec mon texte comme support : on devait remonter le Nil, elle me disait « je vois... je vois les images ». Je crois que lorsqu'elle écrit, c'est ainsi. D'abord elle prend sa source dans l'oralité, elle transcrit, et au moment où elle écrit interviennent aussi des images... lesquelles modifient le cours de l'écrit. Elle construit une sorte de mosaïque, de tableau, en associant des choses qui ne vont pas forcément ensemble. C'est la mise ensemble qui fait apparaitre tout un monde. 
HERVÉ SANSON : J'ai une dernière question, mais en fait tu y as en grande partie répondu, puisque tu as évoqué Les larmes d'Augustin... et la problématique qui se pose à partir de là : est-ce qu'il faut le publier et comment le publier, en quel état...

Mireille Calle-Gruber : Il y a autre chose qu'il faudrait publier et que j'ai... C'est son opéra : Figlie di Ismaele nel vento e la tempesta... Il existe une édition en italien seulement. Elle m'a confié le tapuscrit...

HERVÉ SANSON : donc en français...

Mireille Calle-Gruber : en français, la version originale. Avec l'introduction, écrite aussi par ses soins. J'en ai publié quelques extraits, comme tu sais, dans les actes du Colloque de Cerisy en 2010, et déjà dans l'ouvrage collectif Algérie à plus d'une langue paru au Québec (Études littéraires, 2001). Il faudrait publier l'intégralité.

HeRvÉ SANSON : Et est-ce que tu sais - nous pouvons terminer là-dessus - comment elle a travaillé pour cet opéra ? Parce que moi j'ai en tête évidemment Loin de Médine... Avait-elle son roman sous la main, ou est-ce vraiment quelque chose d'indépendant ?...

Mireille Calle-Gruber : C'est indépendant, et avec d'autres contraintes, celles de la scène, des dialogues, de la musique. Mais il y avait des scènes fondatrices, des élémentsphare que l'on retrouve sous d'autres formes. Et puis c'est elle qui a fait la mise en scène, la scénographie, les costumes... La question de l'image était capitale, c'est-à-dire la question de la représentation, ce qu'on montre et ce que l'on ne montre pas. Le sujet étant religieux, et s'agissant du Prophète, c'était assez épineux. Assia a travaillé avec des masques, joué sur les symboles, la gestuelle, les étoffes, les couleurs... C'est très beau. Le texte était écrit, fixé, elle ne l'a pas changé par rapport à la scénographie, je crois plutôt qu'elle a fait dire autre chose à la scénographie... Elle a amplifié le texte par ses images, qui sont des « tableaux ». Seule la fin n'était pas déterminée, et elle l'a écrite pendant le travail de mise en scène. Convergent ici le travail de l'historienne, celui de la raconteuse, celui de la metteuse en scène. Le questionnement esthétique et méthodologique accompagne la création: il faudrait comme en Italie publier l'ensemble.

HERVÉ SANSON : Justement, nous sommes toujours dans la dernière question, mais je rebondis... Je pensais à l'Italie justement. Elle a quelques pays qui l'ont reconnue, avant la France même, on peut le dire, avec lesquels elle avait un rapport particulier. L'Italie, il me semble, en faisait partie. Et précisément, tu l'as dit, il y a cet opéra qui n'existe pour le moment qu'en italien, et je pense aussi, tu sais bien, à un recueil d'entretiens...

Mireille Calle-Gruber : Oui, qu'elle n'aime pas trop... Elle me les a donnés en français.

HERVÉ SANSON : D'accord. Moi j'ai le livre en italien. C'est juste un exemple, et moi qui vais assez régulièrement en Italie, j'ai constaté que la plupart de ses titres sont traduits en italien, on les trouve dans les librairies, elle a quand même une visibilité en Italie...

Mireille Calle-Gruber : Elle a eu le Prix international de Palmi en 1998, elle a de bonnes traductrices, ce qui compte beaucoup ... Et puis il y a le cinéma, elle a reçu le Prix de la critique internationale à la Biennale de Venise en 1979 pour La Nouba ...

HERVÉ SANSON : Et moi je me souviens des quelques discussions, des quelques entretiens que j'ai pu avoir avec elle, elle m'avait parlé de ce rapport à Venise qu'elle avait... cet italien qu'elle entendait dans les ruelles de Venise et qui la ravissait...

Mireille Calle-Gruber : On en revient au dédale urbain, à la marche fabulante dans la ville... Son opéra Filles d'Ismaël, qui est un drame musical en cinq actes, a été créé à Rome en 2000 avec grand succès. Il a tourné ensuite à Palerme, puis je crois dans le 
nord de l'Italie. Par contre, à l'occasion de l'année de l'Algérie en France, en 2003, nous avons essayé, Assia et moi, de le faire créer à Paris. En vain.

HERVÉ SANSON : On en revient justement à ce que l'on évoquait tout à l'heure: outre les manuscrits, - et au moins on sait que pour le moment ils sont en sécurité chez elle à Paris - en ce qui concerne les films, et leur unique copie, il faut absolument les sauvegarder au plus vite...

Mireille Calle-Gruber : Les sauvegarder, faire faire d'autres copies, mais cela demande des moyens financiers, lesquels en passent par une volonté politique. Personne pour l'instant - ni du côté algérien, ni du côté français - ne s'est investi dans la promotion de l'œuvre cinématographique d'Assia. Actuellement on ne peut pas exploiter ses films en salles, c'est invraisemblable... Nous bricolons avec des $C D$ mais ce n'est pas de très bonne qualité... Pour La Zerda, les sous-titres sont parfois sur le fond blanc. Il y a du travail. Il faut s'y mettre...

HERVÉ SANSON : Je te remercie Mireille, et comme tu l'as dit, il va falloir s'y mettre. Septembre 2015

\section{NOTES}

1. Tombeau d'Akhnaton de Mireille Calle-Gruber a été publié chez La Différence en 2006.

\section{AUTEURS}

\section{MIREILLE CALLE-GRUBER}

Professeur émérite de littérature et d'esthétique à l'Université Paris III-La Sorbonne nouvelle et écrivain. Parmi ses nombreuses publications, celles consacrées à Assia Djebar ont fait d'elle la spécialiste incontestée de cette œuvre. Voici les plus récentes : Assia Djebar, nomade entre les murs... (dir.), chez Maisonneuve \& Larose, 2005 ; Assia Djebar, littérature et transmission (dir.) aux PSN, 2010.

\section{HERVÉ SANSON}

RWTH University / ITEM 